resolved the crystal structure of $\beta$-klotho's extracellular domain when bound to and when free from FGF21, in the absence of FGFRs. Like FGF23, the C-terminal region of FGF21 fits into the groove in $\beta$-klotho. However, the authors could not solve the structure of some regions in $\beta$-klotho, including that corresponding to the RBA in $\alpha$-klotho. This suggests that the RBAs of klotho proteins are intrinsically disordered and unable to fold stably unless bound to FGFRs. The fact that intrinsically disordered proteins can interact with multiple proteins ${ }^{9}$ implies that the RBAs of klotho proteins could capture other partners besides FGFRs. This might explain why the extracellular domain of a-klotho, which can be released into the extracellular space, has been reported to have FGF-independent activity, regulating several ion channels and transporters, along with other growth factors and their receptors ${ }^{5}$.

Another proposed FGF-independent activity for the klothos is as carbohydrate-binding proteins called lectins. Klothos belong to a family of enzymes that cut sugar chains ${ }^{1}$, but not all of the amino-acid residues essential for this enzymatic activity are found in the klothos. Thus, klothos might bind to, but not cut, specific carbohydrates. Lee and colleagues' structure of $\beta$-klotho leaves open the possibility that this protein interacts with particular sugar chains. By contrast, Chen and co-workers' structure of FGF23-a-klotho-FGFR does not fit with the idea of $\alpha$-klotho acting as either an enzyme or a lectin. However, it might be that in the absence of FGFRs, the structure of a-klotho would provide a different point of view. Alternatively, it is possible that the two klothos have different FGF-independent activities.

FGF-klotho signalling has key roles in ageing and age-related disorders. The new structures could be used to develop drugs to treat disorders of ageing, using structurebased drug design to identify targets in FGF-klotho-FGFR complexes. For instance, consider chronic kidney disease $(\mathrm{CKD})^{5,10}-\mathrm{a}$ common state of impaired renal function that often occurs as a complication of high blood pressure or diabetes. People with CKD exhibit many of the same symptoms as mice lacking $a$-klotho, including disturbed phosphate metabolism and increased risk of death ${ }^{5,10}$. Placing mice lacking FGF23 or $\alpha$-klotho on a low-phosphate diet reduces the phosphate retention and premature ageing normally seen in these animals, indicating that phosphate increases accelerate ageing ${ }^{10}$. Thus, drugs that target FGF-klotho-FGFR complexes to improve phosphate metabolism might be useful to treat CKD.

A second example lies in the targeting of FGF21 complexes. FGF21 overexpression extends lifespan in mice $^{11}$, and this protein has been dubbed an 'anti-ageing' hormone. Lee et al. demonstrated that they could increase the potency of FGF21 by introducing genetic mutations designed to increase the protein's affinity for $\beta$-klotho. Further analyses such as this could provide a way to explore anti-ageing medicines more generally.

Makoto Kuro-o is in the Division of Anti-Ageing Medicine, Center for Molecular Medicine, Jichi Medical University, Shimotsuke, Tochigi 329-0498, Japan. e-mail:mkuroo@jichi.ac.jp

1. Kuro-o, M. et al. Nature 390, 45-51 (1997)

2. Kurosu, H. et al. J. Biol. Chem. 281, 6120-6123 (2006).
3. Chen, G. et al. Nature 553, 461-466 (2018).

4. Lee, S. et al. Nature 553, 501-505 (2018).

5. Hu, M. C., Shiizake, K., Kuro-o, M. \& Moe, O. W. Annu. Rev. Physiol. 75, 503-533 (2013).

6. Shimada, T. et al. J. Clin. Invest. 113, 561-568 (2004)

7. Ogawa, Y. et al. Proc. Natl Acad. Sci. USA 104, 7432-7437 (2007).

8. Kurosu, H. et al. J. Biol. Chem. 282, 26687-26695 (2007).

9. Wright, P. E. \& Dyson, H. J. J. Mol. Biol. 293, 321-331 (1999).

10.Kuro-o, M. Nature Rev. Nephrol. 9, 650-660 (2013)

11.Zhang, Y. et al. eLife 1, e00065 (2012).

This article was published online on 17 January 2018.

\title{
A beacon at the dawn of the Universe
}

\section{Quasars are the brightest continuously emitting sources of radiation in the Universe. Measurements of the most distant quasar ever detected reveal details about the evolution and structure of the early Universe. SEE LETTER P.473}

\section{EILAT GLIKMAN}

$\mathrm{S}$ ince their discovery ${ }^{1}$ in 1963 , astronomical objects called quasars have been among our most powerful probes of the early Universe. Initially seen as mysterious sources of extreme luminosity, quasars are now known to be supermassive black holes that are voraciously consuming gas from their immediate surroundings, emitting large amounts of radiation in the process. On page 473, Bañados et al. ${ }^{2}$ report observations of the most distant quasar found so far. The light detected from this object was emitted when the Universe was a mere 690 million years old — just $5 \%$ of its current age.

Almost 90 years ago, the astronomer Edwin Hubble discovered that the Universe is expanding ${ }^{3}$. The expansion stretches light waves travelling through space, such that light that was emitted from a distant source as blue might be detected as red. This phenomenon is called redshift, and is associated with both distance and time: the larger the redshift, the farther away the source was when it emitted its light, meaning that the light was emitted at an earlier time.

If we rewind the expansion, we find that the

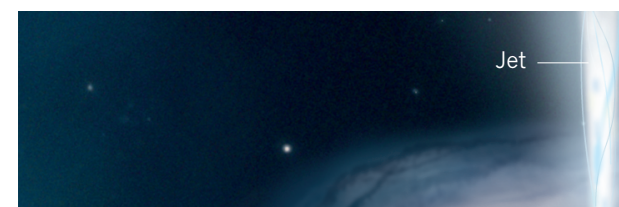

Accretion disk .

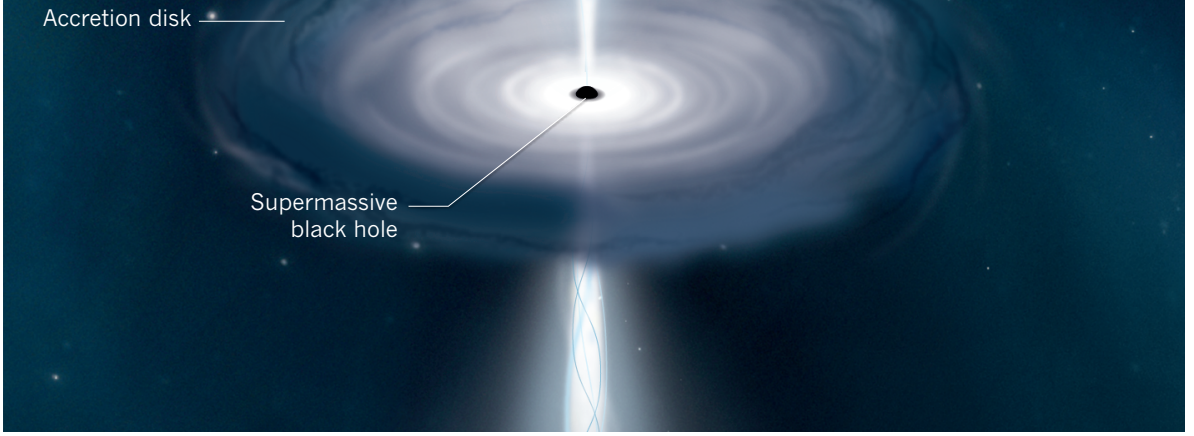

Figure 1 | Emission from a quasar. Quasars are extremely luminous astronomical objects that comprise a supermassive black hole surrounded by an orbiting disk of gas called an accretion disk. As material in the disk is pulled towards the black hole, energy is released in the form of electromagnetic radiation and, in some cases, as beams of charged particles called jets. Bañados et al. ${ }^{2}$ report observations of the most distant quasar identified so far, the light of which was emitted when the Universe was only $5 \%$ of its current age. 
Universe started out in a hot, dense state, filled mostly with ionized hydrogen. As it expanded, it also cooled, and after about 380,000 years, the temperature was low enough for neutral hydrogen to form. For the first few hundred million years, the Universe was devoid of any sources of light - no stars, galaxies or quasars existed. The first stars were then born, but the Universe remained dark because neutral hydrogen is highly effective at absorbing ultraviolet radiation (the main type of emission from these stars).

However, the present-day Universe is filled with sources of light, and the hydrogen that exists in the space between galaxies (the intergalactic medium) is completely ionized and therefore transparent to the ultraviolet emission from early galaxies and quasars. The process of this phase change from a neutral to an ionized Universe, known as reionization, is poorly understood.

The neutral fraction of hydrogen in the Universe can be estimated by analysing the absorption of light by hydrogen in quasars. Studies of quasars observed as they were when the Universe was 0.85 billion to 1.2 billion years old (corresponding to redshifts of 6.5 to about 5 , respectively) have shown that the neutral fraction decreased sharply from $0.1 \%$ to $0.01 \%$ during this time ${ }^{4}$. However, most of the reionization process occurred before this epoch.

Bañados and colleagues' quasar, known as ULAS J1342+0928, has a redshift of 7.54. This means that its strong ultraviolet emission has been shifted into the near-infrared, beyond the sensitivity of typical imaging surveys of the sky. Finding such a high-redshift quasar was not possible until about a decade ago, when sufficiently sensitive near-infrared detectors began scanning large areas of the sky ${ }^{5,6}$. By studying the absorption spectrum of ULAS J1342+0928 (the fraction of incident radiation absorbed by the intergalactic medium over a range of frequencies), the authors determined that the neutral proportion of hydrogen was at least $10 \%$ when the Universe was 690 million years old, which sets a strong constraint on how the intergalactic medium was reionized.

The quasar's black hole is extremely massive - about 800 million times the mass of the Sun. Black holes grow by consuming (accreting) gas from a surrounding structure called an accretion disk (Fig. 1). The gas emits radiation as it falls in. However, such systems have a maximum luminosity, which occurs when the pressure of the emitted light pushes away the infalling gas, halting further growth. This luminosity depends on the mass of the accreting black hole, and therefore defines a maximum growth rate, known as the Eddington limit, for the system.

Bañados et al. suggest that the large mass of the black hole in ULAS J1342+0928 can be explained if the object began its life as an initial (seed) black hole of at least 1,000 solar masses.
This result could rule out models in which black-hole seeds were created from the deaths of the first massive stars ${ }^{7}$, and instead favour models in which these seeds formed from the direct collapse of primordial gas ${ }^{8}$. In addition, the black hole would need to have grown continuously (and, therefore, exponentially) at the Eddington limit, starting from when the Universe was roughly 65 million years old. Although this scenario is physically possible, it requires extreme, sustained accretion for about 600 million years, which is substantially longer than the typical lifetime of a quasar?

So far, only two quasars with redshifts greater than 7 have been discovered. The previous record holder was reported ${ }^{10}$ in 2011, and early models of quasar evolution predicted that more should have been found by now ${ }^{11}$. The methods for finding quasars, even at these high redshifts, are sound and have been proved effective. Therefore, the dearth of high-redshift quasars might indicate that these objects were uncommon in the early Universe, and could imply a sharp decline in quasar activity towards early times ${ }^{12}$. If so, this suggests that we might be observing extremely rare systems as they were beginning to emerge in the Universe.

The authors' work offers a glimpse into the conditions of the intergalactic medium at the earliest epoch of structure formation in the Universe, and could place key constraints on cosmological models of this era. However, a single quasar is insufficient for providing a complete picture of the Universe in the reionization era or of the evolution and growth of supermassive black holes from initial seeds. The task ahead is, then, to mine the upcoming near-infrared sky surveys for additional quasars that can paint a more complete picture of the rapidly evolving early Universe.

Eilat Glikman is in the Department of Physics, Middlebury College, Middlebury, Vermont 05753, USA.

e-mail:eglikman@middlebury.edu

1. Schmidt, M. Nature 197, 1040 (1963).

2. Bañados, E. et al. Nature 553, 473-476 (2018)

3. Hubble, E. Proc. Natl Acad. Sci. USA 15, 168-173 (1929).

4. Fan, X., Carilli, C. L. \& Keating, B. Annu. Rev. Astron. Astrophys. 44, 415-462 (2006).

5. Wright, E. L. et al. Astron. J. 140, 1868-1881 (2010)

6. Lawrence, A. et al. Mon. Not. R. Astron. Soc. 379, 1599-1617 (2007)

7. Natarajan, P. \& Volonteri, M. Mon. Not. R. Astron. Soc. 422, 2051-2057 (2012).

8. Bromm, V. \& Loeb, A. Astrophys. J. 596, 34-46 (2003).

9. Hopkins, P. F. \& Hernquist, L. Astrophys. J. 698, 1550-1569 (2009).

10. Mortlock, D. J. et al. Nature 474, 616-619 (2011)

11. Fan, X. et al. Astrophys. J. 121, 54-65 (2001).

12.McGreer, I. D. et al. Astrophys. J. 768, 105 (2013).

\section{Satellite images show China going green}

Large-scale tree-planting projects have taken place in regions of China prone to soil erosion. Satellite imagery reveals the effects of this work, and shows that a predicted vegetation decline didn't occur during a period of drought.

\section{MARC MACIAS-FAURIA}

$\mathrm{T}$ The effects of human activities on Earth's vegetation have tended to be negative, mostly because of deforestation ${ }^{1}$. Restoration efforts are often restricted to small, localized scales. Large ecological-engineering projects aimed at producing regional-scale effects are few, and among these, China's mega-projects - most notably, the Grain for Green Project (GGP) ${ }^{2}$ - stand out because of their unparalleled scale (27.8 million hectares of forest re-established as of 2013 across 26 Chinese provinces $^{3}$ ). Writing in Nature Sustainability, Tong et al. ${ }^{4}$ report that the positive effects of these tree-planting projects on vegetation growth can be detected using remote-sensing satellite imagery of a large region of southwestern China (the provinces of Guizhou, Guangxi and Yunnan), in an area associated with highly erodible landscapes called karst. The authors note that these projects, which require considerable investment, will be justified only if the modification of ecosystem properties can be achieved on a large scale.

The government-run GGP, intended to halt soil erosion and desertification, began in 1999 (ref. 2). The project's goal was to convert land on mountainous terrain prone to erosion (cropland or scrubland) into forested landscapes (Fig. 1). Such forest would be classified as ecological if trees might eventually be logged (subject to permission) as part of a timber quota, and as economical if it contained orchards, or plantations of trees for medical use. Ecological forest accounted for $80 \%$ of the planting area, with economical forest making up the remaining 20\% (ref. 4). The GGP was developed partly in response to 